\title{
Observation of Quantum Droplets in a Strongly Dipolar Bose Gas
}

\author{
Igor Ferrier-Barbut, Holger Kadau, Matthias Schmitt, Matthias Wenzel, and Tilman Pfau \\ 5. Physikalisches Institut and Center for Integrated Quantum Science and Technology, Universität Stuttgart, \\ Pfaffenwaldring 57, 70550 Stuttgart, Germany \\ (Received 13 January 2016; revised manuscript received 15 February 2016; published 23 May 2016)
}

\begin{abstract}
Quantum fluctuations are the origin of genuine quantum many-body effects, and can be neglected in classical mean-field phenomena. Here, we report on the observation of stable quantum droplets containing $\sim 800$ atoms that are expected to collapse at the mean-field level due to the essentially attractive interaction. By systematic measurements on individual droplets we demonstrate quantitatively that quantum fluctuations mechanically stabilize them against the mean-field collapse. We observe in addition the interference of several droplets indicating that this stable many-body state is phase coherent.
\end{abstract}

DOI: 10.1103/PhysRevLett.116.215301

Uncertainties and fluctuations around mean values are one of the key consequences of quantum mechanics. At the many-body level, they induce corrections to mean-field theory results, altering the many-body state, from a classical factorizable to an entangled state. Owing to their versatility, ultracold atom experiments offer numerous examples of interesting many-body states [1]. Among these systems, bosonic superfluids are well studied. They are described in the weakly interacting regime by a mean-field energy density proportional to the square of the particle density $n^{2}$, with a negative prefactor in the attractive case. Since the seminal work of Lee, Huang, and Yang [2], it is known that interactions lead to a repulsive correction $\propto$ $n^{5 / 2}$ owing to quantum fluctuations. Therefore, an equilibrium between these two contributions can in principle stabilize an attractive Bose gas [3]. A similar stabilization mechanism using quantum fluctuations was proposed for an attractive Bose-Bose mixture in Ref. [4], which leads to the formation of droplets. In this reference liquidlike droplets are defined as the result of a competition between an attractive $n^{2}$ and a repulsive $n^{2+\alpha}$ term in the energy functional. Besides liquid helium droplets [5], such functionals are also used to describe atomic nuclei [6]. Here, we study a strongly dipolar Bose gas where the attractive mean-field interaction is due to the dipole-dipole interaction (DDI). This system is known to be unstable in the mean-field approximation [7]. We however show here that beyond mean-field effects lead to the stabilization of droplets. Our investigations are aimed at probing strongly dipolar Bose gases of ${ }^{164} \mathrm{Dy}$, which are characterized by a dipolar length $a_{\mathrm{dd}}=\mu_{0} \mu^{2} m / 12 \pi \hbar^{2} \simeq 131 a_{0}$, where $a_{0}$ is the Bohr radius with $\mu=9.93 \mu_{B}$ Dy's magnetic dipole moment in units of the Bohr magneton $\mu_{B}, \hbar$ the reduced Planck constant, and $m$ the atomic mass. The additional short-range interaction of ${ }^{164} \mathrm{Dy}$, characterized by the scattering length $a$ has been the focus of several papers [8-11], and the background scattering length was measured to be $a_{\mathrm{bg}}=92(8) a_{0}$, modulated by many Feshbach resonances. Thus, away from Feshbach resonances at the mean-field level the dipolar interaction dominates with $\varepsilon_{\mathrm{dd}, \mathrm{bg}}=a_{\mathrm{dd}} / a_{\mathrm{bg}} \simeq 1.45$. In a previous work [12], we have reported the observation of an instability of a dipolar Bose-Einstein condensate (BEC); the resulting state of this instablity is characterized by the existence of apparent droplets. These droplets cannot be explained by a stabilization by one-body quantum pressure [13], and as such are not solitons in the strict sense.

Here, we isolate these droplets to unravel their nature. To perform our study systematically, we place them in a waveguide. This relaxes their confinement in one direction (along $x$ ) and thus suppresses the effect of dipolar repulsion between the droplets. The waveguide is a single optical dipole trap that creates a tight confinement around the $x$ axis with frequencies $\nu_{y}=123(5) \mathrm{Hz}, \nu_{z}=100(10) \mathrm{Hz}$. The release in this waveguide is performed in the following way (details of the ramping procedures can be found in Ref. [14]): we create a BEC containing $\sim 10 \times 10^{3}$ atoms in a crossed optical dipole trap at a magnetic field along the vertical $(z)$ axis $B_{\mathrm{BEC}}=6.962(10) \mathrm{G}$; we then lower the field to $B_{1}=6.656(10) \mathrm{G}$ in $1 \mathrm{~ms}$, from which a wait time of $15 \mathrm{~ms}$ follows. At $B=B_{1}\left[B=B_{\mathrm{BEC}}\right]$ using $a_{\mathrm{bg}}=92 a_{0}$ and our knowledge of the Feshbach resonances [14], we get $a=95(13) a_{0}\left[a=115(20) a_{0}\right]$. Then, one dipole trap is turned off and the other one ramped-up to higher power in $1 \mathrm{~ms}$. The trap has a too weak confinement to hold the atoms in the $x$ direction and the cloud starts moving. We then image it as function of time in the waveguide $t_{\mathrm{WG}}$ using high-resolution $(1 \mu \mathrm{m})$ imaging. We observe the following, illustrated in Fig. 1. First, the condensed fraction remains fragmented into up to six droplets and down to one droplet. Some atoms originally in the BEC do not form droplets; this fraction of atoms is hard to quantify since it is hard to tell apart from a thermal fraction in our images. Second, during the evolution time the initial confinement energy is turned into relative kinetic energy and these droplets move away from each other. We observe an in situ 

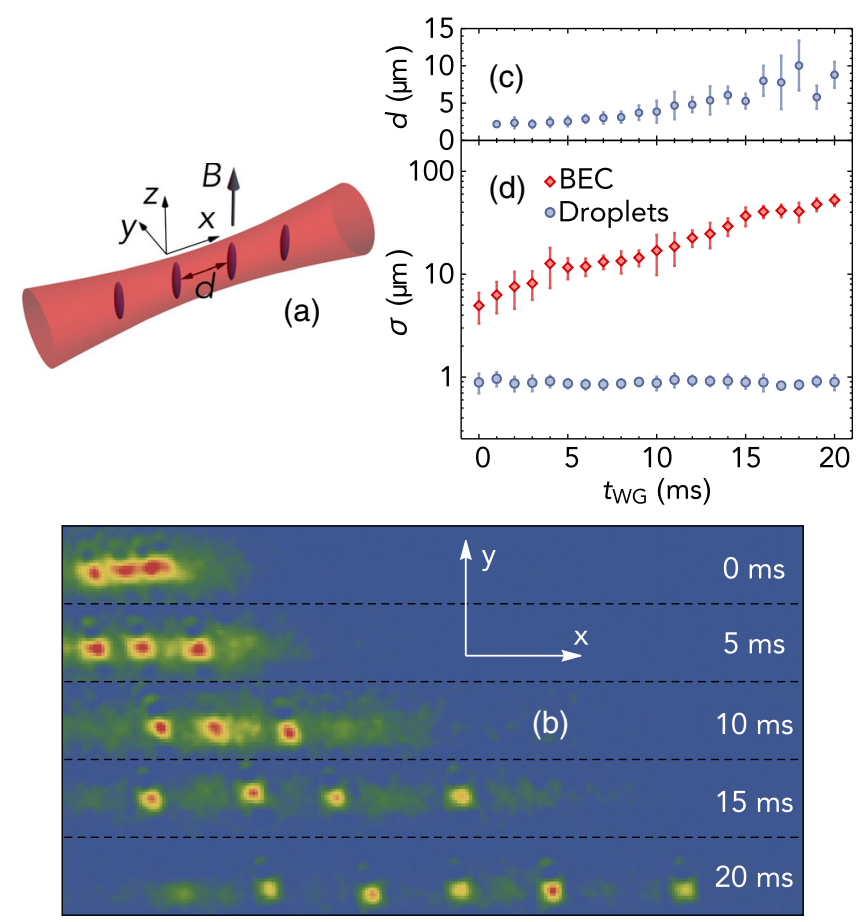

FIG. 1. Quantum droplets of a dipolar Bose gas in a waveguide. (a) Schematic representation of the droplets in the waveguide; the elongation along $z$ is represented; their separation $d$ is indicated. (b) Examples of in situ optical density (OD) images after release in the waveguide at the magnetic field $B_{1}=6.656(10) \mathrm{G}$. Images taken at times $t_{\mathrm{WG}}=0,5,10,15,20 \mathrm{~ms}$ (top to bottom). The OD is normalized to the maximal OD in each image to improve visibility. (c) Evolution of the mean separation $d$ between the droplets as a function of time. (d) Blue circles: evolution of the width $\sigma$ obtained from a Gaussian fit to their density profiles (average of transverse and axial radii). Red diamonds: evolution of the size of a BEC for comparison. The data in panels (c) and (d) are obtained by averaging at least four experimental realizations; the error bars indicate the statistical standard deviation The convention for the axes used through the Letter is indicated in panel (a).

size limited by our resolution (Gaussian width $\sigma \simeq 900 \mathrm{~nm}$ roughly identical in the $x$ and $y$ directions), which does not evolve during $20 \mathrm{~ms}$. If we perform the same sequence but keeping the field at $B_{\mathrm{BEC}}$, we observe that the BEC does not separate into droplets and expands as a whole in the waveguide; in $20 \mathrm{~ms}$ its axial size is increased by a factor 10 [Fig. 1(d), red diamonds]. At $B=B_{1}$, the number of atoms in the droplets is $N=800(200)$. The facts that a single droplet appears to be stable and, when there are several of them, that their size does not significantly increase while their distance is multiplied by 4 , indicates that they are self-confining. Note that we also observe these droplets on the low-field side of a resonance at $B=1.2 \mathrm{G}$.

Since the confinement is too weak in the long direction to observe droplets for longer times, we perform a second set of experiments keeping a very weak confinement in the $x$ direction $\left(\nu_{x}=14.5(1) \mathrm{Hz}\right.$, see Ref. [14]); thus, the trapping potential takes a prolate cigar shape still perpendicular to $\vec{B}$ with an aspect ratio $\nu_{y, z} / \nu_{x} \simeq 8$. We observe that in this trap, the droplets equilibrate at long times $t>100 \mathrm{~ms}$ at an average relative distance $d=2.5(5) \mu \mathrm{m}$, obtained from ten experimental realizations. Furthermore, when we first adiabatically load a BEC in the prolate trap and then ramp from $B_{\mathrm{BEC}}$ to $B_{1}$, we observe the same distance. This distance is smaller than the length obtained by a simple analysis assuming pointlike dipoles in a harmonic trap $l_{x}=\left(3 N \mu_{0} \mu^{2} / 2 \pi m \omega_{x}^{2}\right)^{1 / 5} \simeq$ $4.5 \mu \mathrm{m}$, indicating that the droplets cannot be considered as pointlike. With a more refined analysis developed in Ref. [14] using a Gaussian ansatz with radial symmetry around $z$ for the density distribution inside a droplet, we calculate the dipole-dipole repulsion. We thus obtain that a distance of $d=2.5(5) \mu \mathrm{m}$ is obtained for elongated droplets with $\sigma_{z}=2.5(5) \mu \mathrm{m}$ and a radial size $\sigma_{r} \lesssim 500 \mathrm{~nm}$. Finally, we observe lifetimes of several hundreds of milliseconds, similar to what we reported in Ref. [12], which confirm a strong stabilization mechanism.

Given the strong elongation of the droplets along the $z$ direction, the dipolar interaction is mainly attractive and since $\epsilon_{\mathrm{dd}}=a_{\mathrm{dd}} / a>1$ this attraction is stronger than the short-range repulsion, such that overall the interactions are mainly attractive. The droplets are thus expected to be unstable at the mean-field level [20]. We observe that first the gas locally collapses, before this collapse is arrested at high densities finally forming droplets. This means that the density dependence of the stabilizing mechanism is stronger than that of the mean-field two-body interactions. Importantly, our present work shows that this mechanism is local and not due to any long-range effect between droplets. Two works have postulated the existence of a three-body conservative repulsion [21,22] with mean-field energy density $\propto n^{3}$.

However, these works neglect beyond mean-field effects. As stated above the energy density $e$ for these effects is $e \propto n^{5 / 2}$. This correction has been measured in contactinteracting Bose gases $[23,24]$. Here, we must take both contact repulsion and the DDI into account. Using the results of Refs. [25-27] the beyond mean-field correction to the chemical potential $\mu=(\partial e / \partial n)$ for a dipolar gas is given by $\mu_{\mathrm{bmf}} \simeq(32 g n / 3 \sqrt{\pi}) \sqrt{n a^{3}}\left(1+\frac{3}{2} \epsilon_{\mathrm{dd}}^{2}\right)$, where we have taken the lowest order expansion of the $Q_{5}$ function of Ref. [27] since $\epsilon_{\mathrm{dd}}$ is close to 1 . Doing this we effectively neglect the imaginary part, which is very small compared to the real part, such that a long lifetime is still ensured, though it is only in a metastable equilibrium. This beyond mean-field term is to be compared with the mean-field contact interaction contribution $\mu_{c, \mathrm{mf}}$ and the DDI one $\mu_{\mathrm{dd}, \mathrm{mf}}$. Using a Thomas-Fermi approximation (which neglects kinetic energy) for a droplet with a Gaussian density distribution, the contribution at the center of the droplet is $\mu_{c, \mathrm{mf}}=g n_{0}$ for the contact interaction where 


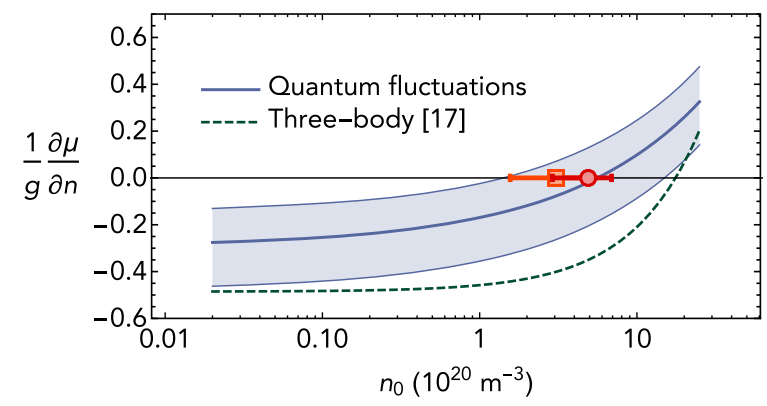

FIG. 2. Derivative of the chemical potential with respect to density as a function of density, at the center of a droplet using a Gaussian ansatz (1) $\left(g=4 \pi \hbar^{2} a / m\right)$. The blue shaded region expresses our uncertainty on the scattering length. Negative values imply mechanical instability. The experimental value obtained from expansion measurements (Fig. 4) is shown as a red circle assuming a Gaussian distribution and as an orange square assuming an inverted parabola. The dashed line shows the same quantity obtained using a three-body repulsion using parameters from Ref. [22], which stabilizes at a higher density.

$g=4 \pi \hbar^{2} a / m$ and $n_{0}$ is the peak density. The dipolar interaction contribution is $\mu_{\mathrm{dd}, \mathrm{mf}}=-g n_{0} \epsilon_{\mathrm{dd}} f_{\mathrm{dip}}(\kappa)$ [28] with $\kappa=\sigma_{r} / \sigma_{z}$; it thus depends on the elongation of the droplet along the field direction through the function $f_{\text {dip }}(\kappa)$, which can be found in Ref. [14]. Using an aspect ratio equal to our experimental upper bound $\kappa=0.2$ one has $f_{\text {dip }}(\kappa)=0.83$ such that the dipolar attraction dominates the mean-field contributions for $\epsilon_{\mathrm{dd}} \geq 1.2$ or $a \leq 110 a_{0}$ [29]. The mechanical stability condition is $(\partial \mu / \partial n) \geq 0$. At the center in the Gaussian ansatz we get

$\left.\frac{\partial \mu}{\partial n}\right|_{\mathbf{r}=0}=g\left(1-\epsilon_{\mathrm{dd}} f_{\mathrm{dip}}(\kappa)+16 \sqrt{n_{0} a^{3} / \pi}\left(1+\frac{3}{2} \epsilon_{\mathrm{dd}}^{2}\right)\right)$,

where $n_{0}$ is the peak density. Note that if one assumes an inverted-parabola density distribution, then one obtains the same result [30]. We plot this function in Fig. 2 using $a=$ 95(13) $a_{0}$ and $\kappa=1 / 10$ [this $\kappa$ value is a factor of 2 below the experimental upper bound; it yields $\left.f_{\text {dip }}(\kappa)=0.94\right]$. One can clearly see that since $\epsilon_{\mathrm{dd}}$ is close to 1, though the attraction dominates, the two mean-field contributions nearly balance each other, which leads to a major role for beyond mean-field effects, a very similar situation to the one considered in Ref. [4]. From Eq. (1) one easily derives that the central density stabilizes at the value

$$
n_{0}=\frac{\pi}{a^{3}}\left(\frac{\epsilon_{\mathrm{dd}} f_{\mathrm{dip}}(\kappa)-1}{16\left(1+3 \epsilon_{\mathrm{dd}}^{2} / 2\right)}\right)^{2}
$$

thus, in our approximation, stability is reached at densities $n_{0} \gtrsim 10^{20} \mathrm{~m}^{-3}$. Equation (2) is striking because the central density does not depend on atom number but only on $a$ and very weakly on $\kappa$ [31], which is characteristic of a liquidlike state. Neglecting quantum fluctuations and assuming a three-body repulsion $\left(\mu_{3 \mathrm{~b}, \mathrm{mf}}=\hbar \kappa_{3} n^{2} / 2\right)$, this density becomes $n_{0}=g\left(\epsilon_{\mathrm{dd}} f_{\text {dip }}(\kappa)-1 / \hbar \kappa_{3}\right)$. Using parameters from Ref. [22] $\left(a=82.6 a_{0}, \kappa_{3}=5.87 \times 10^{-39} \mathrm{~m}^{6} / \mathrm{s}\right)$ we get $n_{0}=17 \times 10^{20} \mathrm{~m}^{-3}$, in very good agreement with full simulation results [22]; at these densities, however, beyond mean-field effects cannot be neglected. In addition such a high value for $\kappa_{3}$ is very hard to justify. It is very probable that $\kappa_{3}$, which is the real part of the three-body coupling constant, lies close to its imaginary part, which is the three-body recombination constant $L_{3}$. Observing the lifetime of the BECs, we have an upper bound $L_{3} \lesssim 10^{-41} \mathrm{~m}^{6} / \mathrm{s}$, which implies an experimentally irrelevant stabilizing density $n_{0}>10^{23} \mathrm{~m}^{-3}$. Our experimental observations developed above imply a lower bound on the central density $n_{0} \geq 10^{20} \mathrm{~m}^{-3}$; given our imaging resolution, we cannot observe smaller droplet radii and higher densities. For a better estimate of the density, we turn to expansion experiments.

The mechanisms at work in the droplets can indeed be further explored by observing their time-of flight expansion in free space. In principle, pure liquid droplets in the absence of trapping should reach an equilibrium with an absence of growth $[4,21]$. On the other hand, time-of-flight expansion under a dipolar interaction is nontrivial but well studied [32,33], and it is modified by beyond mean-field effects [27]; these effects are isotropic and counteract magnetostriction. Mean-field hydrodynamic equations could not describe the expansion of our droplets. In our experiment, we perform such measurements by turning off the waveguide trap after $4 \mathrm{~ms}$. In order to keep the atoms at the focal position of our imaging system, we apply a magnetic field gradient that compensates gravity, and image the atoms at various times after release, Figs. 4(a) and 4(b). We record thus the size in the $x$ and $y$ direction as a function of time. The sizes undergo a linear growth with rates $\dot{\sigma}_{x}=0.17(3) \mu \mathrm{m} / \mathrm{ms}, \dot{\sigma}_{y}=0.24(3) \mu \mathrm{m} / \mathrm{ms}$ [14]. We qualitatively express the expansion dynamics in terms of the released energy $E_{i}=\frac{1}{2} m \dot{\sigma}_{i}^{2}$ [34]; we get $E_{y}=$ $0.09(1) \hbar \omega_{y}, E_{x}=0.045(4) \hbar \omega_{y}$. Such energies are remarkably low, which demonstrates that kinetic energy plays only a marginal role, as expected; however, a full theory is presently not available to describe the free-space dynamics after release.

To circumvent the absence of a model for free-space dynamics, we perform a new set of experiments. It consists of the same procedure, but at the time of release, the magnetic field is quenched (in $50 \mu \mathrm{s}$ ) from $B_{1}$ to a higher value $B_{\text {ToF }}$ inducing a change in scattering length $\Delta a=a\left(B_{\mathrm{ToF}}\right)-a\left(B_{1}\right)$, while the DDI remains unchanged. In this case, the expansion rate is strongly increased. Given the quench time, the initial density does not have time to 


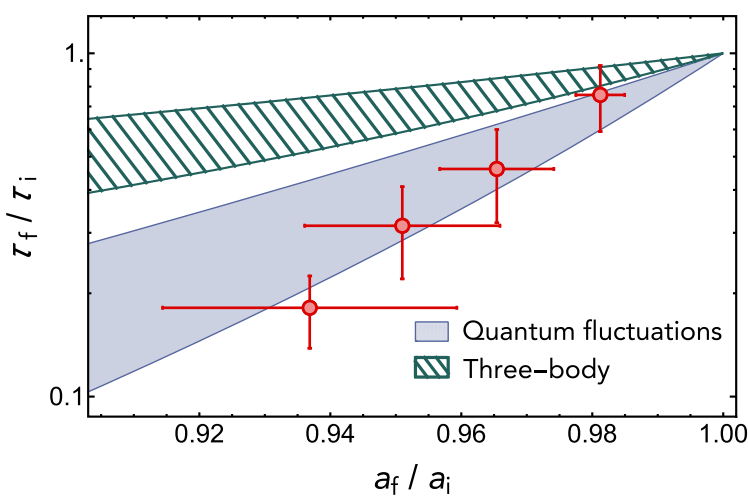

FIG. 3. Ratio of the lifetime $\tau_{f} / \tau i$ of the droplets between scattering lengths $a_{f}$ and $a_{i}$. We use here $a_{i}=94(12) a_{0}$ obtained at $B_{i}=6.573(5) \mathrm{G}$. The data points are taken down to $B_{f}=6.159(5) \mathrm{G}$. The filled blue and green hatched areas represent the expected scaling using quantum fluctuations and three-body repulsion, respectively, taking into account the uncertainty range on the droplets' aspect ratio: $0 \leq \kappa \leq 0.2$.

adapt to the interaction quench. One thus expects that the change in released energy is given by $\Delta E \simeq \frac{1}{N} \int d \vec{r} \frac{\Delta g}{2} n^{2}=$ $\Delta g n_{0} / 4 \sqrt{2}$, where we have used again a Gaussian ansatz, and $\Delta g=4 \pi \hbar^{2} \Delta a / m$. Since we are dealing with the difference in total energy here, the variation of the beyond mean-field corrections is negligible. Thus, since $\Delta a(B)$ is known, we can extract a value for $n_{0}$ from the observed change in $\Delta E$. From these measurements detailed in Ref. [14], given our uncertainty on $a(B)$ we obtain $n_{0}=4.9(2.0) \times 10^{20} \mathrm{~m}^{-3}$. If instead of the Gaussian ansatz we use an inverted parabola, then we get $\Delta E=$ $2 \Delta g n_{0} / 7$, from which we obtain $n_{0}=3.0(1.5) \times 10^{20} \mathrm{~m}^{-3}$. Both values are compatible with the lower bound extracted from in situ imaging; we represent them in Fig. 2. The measured density is thus in agreement with the stabilizing density due to quantum fluctuations.

However, this does not probe the scaling behavior of the density as a function of $a$. As evident in Eq. (2), this scaling is very strong. In turn, three-body recombination in the droplets scales very strongly with $a$; indeed, since the density does not depend on atom number, three-body losses lead to an exponential decay with a lifetime $\tau=1 / L_{3}\left\langle n^{2}\right\rangle$ [14]. In particular, $\tau$ decreases when $a$ decreases. To cancel the uncertainties on $L_{3}$ and on the exact density distribution one simply needs to measure the ratio in lifetime $\tau_{f} / \tau_{i}$ between two different scattering lengths or magnetic fields $B_{i}$ and $B_{f}$, which, assuming a constant $L_{3}$, is simply given by $\tau_{f} / \tau_{i}=\left(\left\langle n_{i}^{2}\right\rangle /\left\langle n_{f}^{2}\right\rangle\right)=\left(n_{0, i}^{2} / n_{0, f}^{2}\right)$. One can easily show that for a fixed $\kappa$ this ratio is a function of only two parameters $\left[\left(a_{f} / a_{i}\right),\left(a_{\mathrm{dd}} / a_{i}\right)\right]$; in particular assuming three-body repulsion, it is independent of $\kappa_{3}$; we give this function in Ref. [14]. Thus, using a fixed $a_{i}=94(12) a_{0}$ $\left[B_{i}=6.573(5) \mathrm{G}\right]$, in Fig. 3 we represent $\tau_{f} / \tau_{i}$ vs $a_{f} / a_{i}$. This figure is striking; while we vary the scattering length

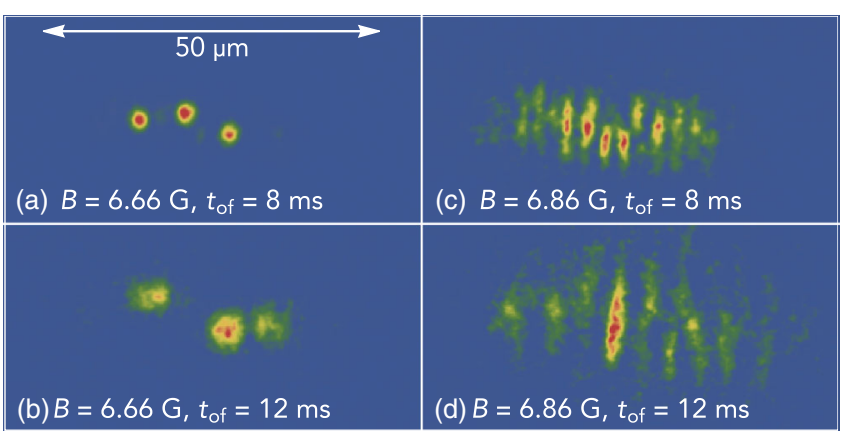

FIG. 4. Time-of-flight expansion measurements. The field is held at $B_{1}=6.656(10) \mathrm{G}$ until release when it is quenched to a different value. (a),(b) Images where the field is kept at $B_{1}$ during expansion and (c),(d) quenched to $6.86 \mathrm{G}$. In (a) and (b) one sees expanding droplets, whereas in (c) and (d) they overlap and clear interference fringes appear along the $x$ axis while we can still measure the expansion size in the $y$ direction.

by less than $10 \%$, the lifetime is divided by a factor of 5 . Furthermore, the data points are incompatible with the scaling predicted by three-body repulsion while without any fit parameter they follow the scaling predicted using quantum fluctuation within the experimental uncertainties. The small deviation to lower lifetimes can be accounted for by a weak variation of $L_{3}$ [35]. This demonstrates unambiguously that quantum fluctuations constitute the stabilizing mechanism. The conclusion we drew here is reinforced by numerical simulations reported in [36] of which we have recently become aware. Finally, we observe that the droplets have internal phase coherence. Indeed, for "fast" expansion dynamics obtained when quenching $B$ during the time of flight, the size of the expanding droplets becomes comparable to or larger than their relative distance so that neighboring ones overlap. In this case we observe matter-wave interference fringes as exemplified in Figs. 4(c) and 4(d). The presence of these fringes demonstrates that each droplet individually is phase coherent and thus superfluid. Their observation opens the door to studies of the relative phase coherence between droplets. In the present case we do not observe fringe patterns that allow us to measure the droplets' relative phase, but this is mainly due to shot-to-shot noise in the in situ position and the relative spacing of the droplets since we are not yet in the far-field regime. Future studies with fixed in situ conditions prior to the time of flight could bring insight into the phase coherence of an ensemble of droplets, even in the case of a high number of them [37]. Our measurements reported here have established the existence of a novel system forming droplets stabilized by quantum fluctuations. These results open prospects of forming pure liquid droplets of a quantum gas in free space characterized by a total absence of growth.

We acknowledge insightful discussions with L. Santos and D. Petrov as well as with H.P Büchler, A. Pelster, 
G. Raithel, and F. Ferlaino. We thank T. Maier for experimental assistance at early stages. This work is supported by the German Research Foundation (DFG) within SFB/TRR21.

[1] I. Bloch, J. Dalibard, and W. Zwerger, Many-body physics with ultracold gases, Rev. Mod. Phys. 80, 885 (2008).

[2] T. D. Lee, K. Huang, and C. N. Yang, Eigenvalues and eigenfunctions of a bose system of hard spheres and its low-temperature properties, Phys. Rev. 106, 1135 (1957).

[3] G. E. Volovik, The Universe in a Helium Droplet, International Series of Monographs on Physics (Oxford University Press, Oxford, 2009).

[4] D.S. Petrov, Quantum Mechanical Stabilization of a Collapsing Bose-Bose Mixture, Phys. Rev. Lett. 115, 155302 (2015).

[5] F. Dalfovo, A. Lastri, L. Pricaupenko, S. Stringari, and J. Treiner, Structural and dynamical properties of superfluid helium: A density-functional approach, Phys. Rev. B 52, 1193 (1995).

[6] M. Bender, P.-H. Heenen, and P.-G. Reinhard, Selfconsistent mean-field models for nuclear structure, Rev. Mod. Phys. 75, 121 (2003).

[7] S. Komineas and N. R. Cooper, Vortex lattices in BoseEinstein condensates with dipolar interactions beyond the weak-interaction limit, Phys. Rev. A 75, 023623 (2007).

[8] K. Baumann, N. Q. Burdick, M. Lu, and B. L. Lev, Observation of low-field Fano-Feshbach resonances in ultracold gases of dysprosium, Phys. Rev. A 89, 020701 (R) (2014).

[9] T. Maier, I. Ferrier-Barbut, H. Kadau, M. Schmitt, M. Wenzel, C. Wink, T. Pfau, K. Jachymski, and P. S. Julienne, Broad universal Feshbach resonances in the chaotic spectrum of dysprosium atoms, Phys. Rev. A 92, 060702(R) (2015).

[10] Y. Tang, A. Sykes, N. Q. Burdick, J. L. Bohn, and B. L. Lev, $s$-wave scattering lengths of the strongly dipolar bosons ${ }^{162}$ Dy and ${ }^{164}$ Dy, Phys. Rev. A 92, 022703 (2015).

[11] T. Maier, H. Kadau, M. Schmitt, M. Wenzel, I. FerrierBarbut, T. Pfau, A. Frisch, S. Baier, K. Aikawa, L. Chomaz, M. J. Mark, F. Ferlaino, C. Makrides, E. Tiesinga, A. Petrov, and S. Kotochigova, Emergence of Chaotic Scattering in Ultracold Er and Dy, Phys. Rev. X 5, 041029 (2015).

[12] H. Kadau, M. Schmitt, M. Wenzel, C. Wink, T. Maier, I. Ferrier-Barbut, and T. Pfau, Observing the Rosensweig instability of a quantum ferrofluid, Nature (London) $\mathbf{5 3 0}$, 194 (2016).

[13] P. Pedri and L. Santos, Two-Dimensional Bright Solitons in Dipolar Bose-Einstein Condensates, Phys. Rev. Lett. 95, 200404 (2005)

[14] See Supplemental Material at http://link.aps.org/ supplemental/10.1103/PhysRevLett.116.215301 for a detailed description of the different magnetic field and trapping lasers ramping procedures as well as a presentation of the theoretical models used throughout the Letter, which includes Refs. [15-19].
[15] K. Jachymski and P. S. Julienne, Analytical model of overlapping Feshbach resonances, Phys. Rev. A 88, 052701 (2013).

[16] M. Fattori, G. Roati, B. Deissler, C. D’Errico, M. Zaccanti, M. Jona-Lasinio, L. Santos, M. Inguscio, and G. Modugno, Magnetic Dipolar Interaction in a Bose-Einstein Condensate Atomic Interferometer, Phys. Rev. Lett. 101, 190405 (2008).

[17] B. Gao, General form of the quantum-defect theory for $-1 / r^{\alpha}$ type of potentials with $\alpha>2$, Phys. Rev. A 78, 012702 (2008).

[18] Y. Wang and P. S. Julienne, Universal van der Waals physics for three cold atoms near Feshbach resonances, Nat. Phys. 10, 768 (2014).

[19] Z. Shotan, O. Machtey, S. Kokkelmans, and L. Khaykovich, Three-Body Recombination at Vanishing Scattering Lengths in an Ultracold Bose Gas, Phys. Rev. Lett. 113, 053202 (2014).

[20] T. Koch, T. Lahaye, J. Metz, B. Fröhlich, A. Griesmaier, and T. Pfau, Stabilization of a purely dipolar quantum gas against collapse, Nat. Phys. 4, 218 (2008).

[21] K.-T. Xi and H. Saito, Droplet formation in a Bose-Einstein condensate with strong dipole-dipole interaction, Phys. Rev. A 93, 011604 (2016).

[22] R. N. Bisset and P. B. Blakie, Crystallization of a dilute atomic dipolar condensate, Phys. Rev. A 92, 061603(R) (2015).

[23] S. B. Papp, J. M. Pino, R. J. Wild, S. Ronen, C. E. Wieman, D. S. Jin, and E. A. Cornell, Bragg Spectroscopy of a Strongly Interacting ${ }^{85} \mathrm{Rb}$ Bose-Einstein Condensate, Phys. Rev. Lett. 101, 135301 (2008).

[24] N. Navon, S. Piatecki, K. Günter, B. Rem, T. C. Nguyen, F. Chevy, W. Krauth, and C. Salomon, Dynamics and Thermodynamics of the Low-Temperature Strongly Interacting Bose Gas, Phys. Rev. Lett. 107, 135301 (2011).

[25] R. Schützhold, M. Uhlmann, Y. Xu, and U. R. Fischer, Mean-field expansion in bose-einstein condensates with finite-range interactions, Int. J. Mod. Phys. B 20, 3555 (2006).

[26] A. R. P. Lima and A. Pelster, Quantum fluctuations in dipolar Bose gases, Phys. Rev. A 84, 041604(R) (2011).

[27] A. R. P. Lima and A. Pelster, Beyond mean-field low-lying excitations of dipolar Bose gases, Phys. Rev. A 86, 063609 (2012).

[28] T. Lahaye, C. Menotti, L. Santos, M. Lewenstein, and T. Pfau, The physics of dipolar bosonic quantum gases, Rep. Prog. Phys. 72, 126401 (2009).

[29] Since $f_{\text {dip }}(\kappa)$ is a monotonically decreasing function of $\kappa$, at lower aspect ratios than 0.2 , the mean-field dipolar contribution is larger and renders the droplets more unstable in the mean-field approximation.

[30] D. H. J. O'Dell, S. Giovanazzi, and C. Eberlein, Exact Hydrodynamics of a Trapped Dipolar Bose-Einstein Condensate, Phys. Rev. Lett. 92, 250401 (2004).

[31] At our aspect ratios $f_{\text {dip }}(\kappa)$ depends only weakly on $\kappa$ as is visible in the Supplemental Material [14].

[32] S. Giovanazzi, P. Pedri, L. Santos, A. Griesmaier, M. Fattori, T. Koch, J. Stuhler, and T. Pfau, Expansion dynamics of a dipolar Bose-Einstein condensate, Phys. Rev. A 74, 013621 (2006). 
[33] T. Lahaye, T. Koch, B. Fröhlich, M. Fattori, J. Metz, A. Griesmaier, S. Giovanazzi, and T. Pfau, Strong dipolar effects in a quantum ferrofluid, Nature (London) 448, 672 (2007).

[34] M. J. Holland, D. S. Jin, M. L. Chiofalo, and J. Cooper, Emergence of Interaction Effects in Bose-Einstein Condensation, Phys. Rev. Lett. 78, 3801 (1997).
[35] Measurements of $L_{3}$ will be presented in a future publication.

[36] F. Wächtler and L. Santos, Quantum filaments in dipolar Bose-Einstein condensates, arXiv:1601.04501.

[37] Z. Hadzibabic, S. Stock, B. Battelier, V. Bretin, and J. Dalibard, Interference of an Array of Independent BoseEinstein Condensates, Phys. Rev. Lett. 93, 180403 (2004). 